\title{
Hot topics and cold lecture halls: the ECCMID 2019
}

\author{
Johannes R. Bogner ${ }^{1}$ \\ Published online: 25 April 2019 \\ (c) Springer-Verlag GmbH Germany, part of Springer Nature 2019
}

Again, the ECCMID was a big success for the organisers as well as for roughly 13,500 participants from 120 countries: infectious disease clinicians, microbiologists, researchers, and representants of diagnostic and therapeutic industrial manufacturers. The scientific programme according to the programme director Winfried V. Kern was composed from 5311 regular scientific abstract submissions and additional 142 late-breaker submissions originating from more than 100 countries. In addition to Paper-, Poster- and e-Poster Sessions, 132 Oral Talk sessions were presented, most of them simultaneously.

One of the symposia (Sy 010) was dedicated to the management of chronic bone and joint infections. Because it is not always possible to perform necessary surgical interventions on patients with chronic prosthetic joint infections due to individual reasons (age, co-morbidity, patients decision), we end up in situations where long-term suppressive antibiotic treatment becomes necessary-a topic that has not been extensively studied in clinical trials [1, 2]. However, this approach is controversial and therefore it was the aim to talk about alternative and promising options to rescue patients without surgery, to include novel anti-persister antibiotics, and to use local antibiotic delivery and phage therapy [2].

Marjan Wouthuyzen-Bakker reported on suppressive antibiotic treatment for peri-prosthetic joint infections (PJP) which may be successful over months and sometimes even years. Her presentation was followed by that of Ho K. Li, who presented data of the recently published OVIVA trial from the United Kingdom. In addition to the main outcome of non-inferiority of the oral treatment, he also reported on a significant difference in adverse events caused by linecomplications and a difference in cost that amounts to an average of 2740 British Pounds per patient saved [3]. Tristan Ferry talked about novel anti-persister antibiotics, local

Johannes R. Bogner

jbogner@med.lmu.de

1 Sektion Klinische Infektiologie, Med. Klinik und Poliklinik IV, Klinikum der Universität München, Munich, Germany antibiotic delivery and phage therapy as possible innovative treatments. As persister-organisms escape the effects of antibiotics by intracellular persistence and biofilm formation, new options can be subcutaneous delivery of ceftazidime, cerament releasing high local concentrations of gentamicin and even application of bacteriophage therapy. Overall, like other sessions this one was overcrowded with many participants outside Hall D in an overflow area watching the presentations on a screen and hearing via headphones which were provided by the congress organisation-another example how well organised this conference could react to the higher than expected number of participants for this session.

One keynote lecture and several other contributions were dedicated to results of microbiome research (KN 077) [4]. Trevor Lawley started with the equation "Visible Organs + Invisible Microbiome = Complete Human". He explained how modern lifestyles are damaging our microbiomes. Dysbiosis in gut microbiome impairs human health and transmission of commensal intestinal bacteria between humans could promote health by establishing, maintaining and replenishing microbial diversity in the microbiota of an individual. He presented results showing that sporulation is a fundamental feature and that it is an evolving property of human gut microbiota. The formation of the human ecosystem is also dependent on development during early life. He pointed at highly significant differences in microbiome composition and abundance between vaginal delivery and caesarean section delivery.

The big question is how this difference affects health and disease development in later life-even after childhood and adolescence. This topic was also addressed with results of Martha Reyman (OS041): her study suggests that the mode of delivery influences the development of the microbial composition of the gut microbiota. This, in turn, may affect infants' respiratory health during the first year of life. The study included 120 infants and found that children born by caesarean section had a delay in the normal development of their gut microbiota, and a higher abundance of potentially harmful bacteria, compared to infants delivered vaginally. This could be the mechanism driving the increased risk in 
respiratory infections. "The gut microbiota was less stable and the development of the health-promoting bacterial species Bifidobacterium spp. was delayed compared to vaginally delivered children. Additionally, infants born by C-section had much higher levels of potential pathogenic gut bacteria, irrespective of length of hospital stay after birth, feeding type, and antibiotic use."

Kiran R. Patel reported on experiments looking at the inhibition of commensal gut bacteria after application of non-antibiotic drugs. He found that about one-quarter of all investigated drugs (non-antibiotics, e.g., antidepressants, e.g., duloxetine or anti-diabetics) lead to a significant alteration of the gut microbiome. Observed effects were biotransformation, bioaccumulation, and growth inhibition as opposed to growth enhancement [5].

Ron Dagan gave a wise review on changes of the nasopharyngeal microbiome as caused by pneumococcal vaccinations [6]. Pneumococci of different subtypes compete with each other and compete with other flora components (e.g., Haemophilus, Moraxella). Pneumococcal conjugate vaccines (PCV) also influence the nasopharyngeal microbiome and lead to changes in serotype composition. And this can have influence on the incidence of disease due to Haemophilus influenza. Moreover, response to RSV seems to be different depending on composition of nasopharyngeal microbiota and vaccination status. "The pneumococcal vaccine is acting on the whole zoo" of the nasopharyngeal microbiota.

In terms of upcoming new antibacterials, there was an abundance of poster presentations showing that the preclinical end early clinical development of new compounds mainly of the betalactamase-inhibitor type is ongoing. Examples are meropenem/nacubactam, enmetazobactam, cefepime/WCK5222, cefepime/VNRX-5133, ceftibuten/ VNRX-7145 (orally available), cefpodoxim/ETX1317 and Sulbactam/ETX2514.

However, also alternative new concepts dealing with antibiotic development were discussed in Symposium 056: Paula Gameiro talked about bioinorganic antimicrobial platforms, metal-modified macromolecules, metal and metal oxides as well as nanoparticles: ternary complexes of new fluoroquinolones including a copper ion might be able to overcome resistance development and achieve high antibacterial activity. Gaëtan Mislin talked about development and vectorisation of innovative organometallics against pathogenic bacteria: TMB3 is a betalactam including a gold molecule which leads to an increase of Gram-positive activity while toxicity is in an acceptable therapeutic window (in vitro). And Marvin Miller gave a fascinating overview on siderophore antibacterials using the "Trojan horse" strategy to enter the bacterial cell.

Sy067 brought together specialists in S. aureus bacteremia in order to discuss the question whether combination therapy may be superior to monotherapy [7, 8]. Annelies Zinkernagel very clearly pointed at the impossibility of eradicating Staphylococci from biofilms and difficulties with resistance development of bacteria in the biofilm. Miquel Pujol came to the conclusion that for patients with implants and with MRSA, combination therapy was superior.

In Sy 103 (Climate changes and infectious diseases) Giovanni Rezza reported about an autochthonous outbreak of Chikungunya virus near Ravenna, Italy, in 2007 [9]. This was brought to the region by a traveller from India who fell sick upon arrival. Obviously this individual must have been bitten by a mosquito which was able to further distribute the virus locally. Ten years later, in 2017, another outbreak occurred in Anzio and Rome and there was a secondary outbreak in Calabria. This is only one example for changing climate effects on epidemiology of tropical diseases. However, discussions about climate changes towards global warming took place in this hall at freezing temperatures produced by an obviously over-active air conditioning, which resulted in most ECCMID visitors appearing in their coats in order not to catch a cold at the infectious disease meeting while the sun was shining outside.

Also some unexpected results were presented at ECCMID: who would have thought that staying at a hotel may be more dangerous in terms of becoming colonised with resistant organisms as compared to other accommodations? Staying in a hotel or private house is associated with contracting and carrying home drug-resistant bacteria in travellers to low and/or middle-income countries, according to new research by Lynn Meurs together with Christoph Lübbert (Poster PS100). In a study comprising 230 young travellers from Germany those who mainly stayed in a hotel or private accommodation had a four times higher risk in each case of returning home with multi-drug resistant bacteria in their gut than those who mainly stayed in other types of tourist accommodations like guest houses, hostels, or camping. According to the authors, the study is the first to report staying in a hotel as a risk factor for colonisation with ESBL organisms.

The researchers collected stool samples from participants before and after they travelled outside Germany. All travellers completed questionnaires on risk factors including the countries they visited, length of time in country, type of residence, symptoms, antibiotic treatment, healthcare use, diet, and hygiene. Results showed that around one in five travellers $(23 \%$; 53/230) contracted ESBL-producing bacteria during their trip abroad. A journey to Western, Southern or Eastern Asia was associated with the highest risk of contracting the resistant bacteria (four times higher than tropical Africa). The authors comment: "This observational study in one travel clinic cannot prove that the type of accommodation causes colonisation with ESBL-producing bacteria, but only suggests the possibility of such an effect". They also 
point to several limitations including that the study was not sufficiently powered to detect other risk or protective factors for travel-associated ESBL acquisition and that travellers attending a travel clinic may not be representative for all people travelling to the tropics. Christoph Lübbert's group is already known for his unexpected or sensational results since he reported on antimicrobials and resistant organisms in water probes in the proximity of Indian pharmaceutical production plants [10].

\section{Compliance with ethical standards}

Conflict of interest The author has no conflict of interest regarding any antimicrobial agents and industry developing those.

\section{References}

1. Pradier M, Robineau O, Boucher A, et al. Suppressive antibiotic therapy with oral tetracyclines for prosthetic joint infections: a retrospective study of 78 patients. Infection. 2018;46:39-47.

2. Staats K, Sevelda F, Kaider A, et al. The influence of antibioticloaded cement spacers on the risk of reinfection after septic twostage hip revision surgery. Infection. 2017;45:885-91.
3. Li HK, Rombach I, Zambellas R, et al. Oral versus intravenous antibiotics for bone and joint infection. N Engl J Med. 2019;380:425-36.

4. Pham TA, Lawley TD. Emerging insights on intestinal dysbiosis during bacterial infections. Curr Opin Microbiol. 2014;17:67-74.

5. Maier L, Pruteanu M, Kuhn M, et al. Extensive impact of nonantibiotic drugs on human gut bacteria. Nature. 2018;555:623-8.

6. Weinberger DM, Pitzer VE, Regev-Yochay G, Givon-Lavi N, Dagan R. Association between the decline in pneumococcal disease in unimmunized adults and vaccine-derived protection against colonization in toddlers and preschool-aged children. Am J Epidemiol. 2019;188:160-8.

7. Forsblom E, Kakriainen A, Ruotsalainen E, Jarvinen A. Comparison of patient characteristics, clinical management, infectious specialist consultation, and outcome in men and women with methicillin-sensitive Staphylococcus aureus bacteremia: a propensity-score adjusted retrospective study. Infection. 2018;46:837-45.

8. Jung N, Rieg S. Essentials in the management of $S$. aureus bloodstream infection. Infection. 2018;46:441-2.

9. Lindh E, Argentini C, Remoli ME, et al. The Italian 2017 outbreak chikungunya virus belongs to an emerging Aedes albopictus-adapted virus cluster introduced from the indian subcontinent. Open Forum Infect Dis. 2018;6(1):ofy321. https://doi. org/10.1093/ofid/ofy321

10. Lubbert C, Baars C, Dayakar A, et al. Environmental pollution with antimicrobial agents from bulk drug manufacturing industries in Hyderabad, South India, is associated with dissemination of extended-spectrum beta-lactamase and carbapenemase-producing pathogens. Infection. 2017;45:479-91. 